\title{
Prognostic significance of osteopontin in acute myeloid leukemia: A meta-analysis
}

\author{
YONG-BING CHEN ${ }^{1}$, SI-MEI REN ${ }^{2}$, SI-DAN LI ${ }^{3}$ and ZHONGLI DU ${ }^{2}$ \\ ${ }^{1}$ Department of Hepatobiliary Surgery, PLA Army General Hospital, Beijing 100700; ${ }^{2}$ Department of Hematology/National \\ Center for Clinical Laboratories, Beijing Hospital, Beijing 100730; ${ }^{3}$ Beijing Key Laboratory of Pediatric Hematology \\ Oncology/National Key Discipline of Pediatrics, Ministry of Education/Key Laboratory of Major Diseases in Children, \\ Ministry of Education/Hematology Oncology Center, Beijing Children's Hospital, \\ Capital Medical University, Beijing 100045, P.R. China
}

Received August 30, 2016; Accepted May 22, 2017

DOI: $10.3892 / \mathrm{mco} .2017 .1302$

\begin{abstract}
Osteopontin (OPN) has been investigated in the field of tumor research for several years. However, the prognostic role of OPN overexpression in acute myeloid leukemia (AML) remains controversial. A meta-analysis of four studies, including a total of 492 patients, was performed to determine the association of OPN with overall survival (OS) in AML patients. The random-effects model of Der Simonian and Laird was used to synthesize data; hazard ratio (HR) with its $95 \%$ confidence interval (CI) was used as the effect size estimate. It was observed that serum-based OPN was inversely correlated with OS and the difference was statistically significant $(\mathrm{HR}=1.83$; 95\% CI: 1.43-2.35; $\mathrm{P}<0.001)$. Experimental findings indicate that $\mathrm{OPN}$ overexpression is associated with a poor prognosis in AML and may be of prognostic value for AML stage and metastasis.
\end{abstract}

\section{Introduction}

Acute myeloid leukemia (AML) is a unique type of malignancy, the main treatment for which is chemotherapy alone or chemotherapy combined with radiotherapy, as surgical intervention is not indicated, with certain exceptions (1). Although significant progress has been made in the diagnosis and treatment of AML, the prognosis of leukemia patients is dismal due to recurrence and poor response to adjunctive treatments in advanced stages. Thus, it is crucial to identify predictive

Correspondence to: Dr Si-Dan Li, Beijing Key Laboratory of Pediatric Hematology Oncology/National Key Discipline of Pediatrics, Ministry of Education/Key Laboratory of Major Diseases in Children, Ministry of Education/Hematology Oncology Center, Beijing Children's Hospital, Capital Medical University, 56 Nanlishi Road, Beijing 100045, P.R. China

E-mail: lisidan2006@126.com

Key words: osteopontin, prognosis, acute myeloid leukemia, meta-analysis factors for prognosis and metastasis/recurrence, which may help with regimen selection and improve patient survival.

Predictive factors are subdivided into host-related (e.g., age and performance status) and tumor-related (e.g., number of tumor cells, cytogenetic and molecular characteristics of the leukemic clone, growth characteristics and resistance to apoptosis). Hematological malignancies are not only affected by the molecular interactions within the malignant cell clone, but also by interactions with the microenvironment. The association between niche cells and malignant cells significantly affects the pathophysiological behavior and development of the disease. Leukemic cell localization in the bone marrow and the cross-talk with the bone niche trigger marked changes in the bone marrow microenvironment, which are critical for tumor progression, resistance to treatment and recurrence (2-5).

Osteopontin (OPN) is a candidate prognostic factor for the progression of various hematological malignancies. OPN was initially considered to be a transformation-associated protein in the epithelial cells (6) and has since been extensively investigated. OPN is a secreted glycophosphoprotein that may physiologically act as a cytokine as well as an extracellular matrix molecule. A number of studies recently demonstrated that overexpression of OPN is significantly associated with tumorigenesis and metastasis in several malignant tumors, such as breast, pulmonary, prostatic, thyroid, ovarian, bladder and gastrointestinal carcinomas (7-9). Furthermore, aberrant OPN expression has been correlated with poor prognosis of leukemia (10-12).

Although a number of studies have considered OPN as a marker in patient specimens, published data have not yet been fully analyzed. Moreover, conflicting results have been reported regarding the value of OPN as predictive factor for overall survival (OS) in hematological malignancies. Thus, a meta-analysis is required in order to systematically and comprehensively elucidate the prognostic value of OPN in hematological malignancies.

\section{Data collection methods}

The aim of the present study was to objectively evaluate the prognostic role of elevated OPN levels for OS in patients with AML using pooled results from the available data. 
Criteria for study inclusion. This systematic review was performed according to the Preferred Reporting Items for Systematic Reviews and Meta-Analyses (PRISMA) and the Cochrane Handbook for Systematic Reviews of Interventions (http://handbook.cochrane.org/).

Identification of studies. A search was conducted through PubMed, China National Knowledge Infrastructure, Web of Science, Embase and the Cochrane Central Register of Controlled Trials from inception until December 25th, 2015, and the articles referenced in the identified studies were also manually searched for additional studies that met the inclusion criteria. No restriction on publication status was applied. The search terms included osteopontin (1-168) human or SPP1 protein human (13).

Data collection and analysis. Two reviewers (Drs Sidan Li and Yongbing Chen) independently screened the initially identified studies. The full-text articles of potentially eligible studies were independently assessed against the eligibility criteria. Differences were then compared and referred to the consultants (Drs Zhongli Du and Simei Ren) for resolution. When more than one article was derived from the same study, only the most eligible records were included, considering data integrity and availability.

Selection of studies. The inclusion criteria were as follows: i) Measurement of OPN expression in patients with leukemia, lymphoma or myeloma by reverse transcription quantitative polymerase chain reaction (RT-qPCR), enzyme-linked immunosorbent assay (ELISA), microarray analysis or immunohistochemistry (IHC); ii) investigation of the possible correlation between changes in OPN expression and clinical prognosis; and iii) information on survival.

Data extraction and management. Two researchers (Dr Sidan Li and Dr Yongbing Chen) independently extracted data and selected the included studies. If there was discrepancy in data selection, the two researchers would review and discuss the study together, or resort to the consultants (Dr Zhongli Du and Dr Simei Ren) until a consensus was reached. When there more than one article was derived from the same study, only the most eligible records were included, considering data integrity and availability. The main reasons for exclusion of trials are described in Fig. 1.

Definitions. In the meta-analysis, OS was defined as the time from the diagnosis of hematological malignancies or initiation of different medical treatments to death or the date of the last follow-up.

Quality assessment. Quality assessment of available data included in this meta-analysis on the significance of OPN in hematological malignancies was performed independently by the two researchers in accordance with the Newcastle-Ottawa Scale (NOS) for cohort studies and case control studies, with mild modifications (13). This scale assesses patient population and selection, study comparability, follow-up and outcome of interest with scores ranging from 0 (lowest quality) to 9 (highest quality). The high-quality studies had scores of $\geq 6$.
Table I. Assessment of methodological quality according to Newcastle-Ottawa Scale quality assessment.

\begin{tabular}{lcccc}
\hline Author & Selection & Comparability & Outcome & (Refs.) \\
\hline Liersch et al & $* * * *$ & $* *$ & $* * *$ & $(12)$ \\
Liersch et al & $* * * *$ & $* *$ & $* *$ & $(12)$ \\
Liersch et al & $* * * *$ & $* *$ & $* *$ & $(12)$ \\
Powell et al & $* * * *$ & $* *$ & $*$ & $(11)$ \\
Lee et al & $* * * *$ & $* *$ & $*$ & $(14)$ \\
\hline
\end{tabular}

Statistical analysis. Survival outcome data were synthesized with the hazard ratio (HR) and its $95 \%$ confidence interval (CI) to analyze the effect of OPN expression on the survival of hematological malignancies. Studies providing HRs and 95\% CIs were pooled directly. Otherwise, the HR and its 95\% CI were calculated from available data or a Kaplan-Meier survival curve using Engauge Digitizer software, version 4.1 (http://sourceforge.net). The heterogeneity among the data was assessed by a Chi-squared-based Q statistic test, and the $\mathrm{I}^{2}$ value was used to quantify the heterogeneity $\left(\mathrm{I}^{2}=0-50 \%\right.$, no or moderate heterogeneity; $\mathrm{I}^{2}>50 \%$, significant heterogeneity). If homogeneity was not significant ( $\mathrm{Q}$ test $\mathrm{P}>0.10$ ), the fixed-effects model was used; otherwise, the random-effects model was applied. Publication bias was assessed using the Begg's and Egger's tests. To adjust for multiple comparisons, the stepdown Bonferroni method was applied, which controls for family-wise error rate. Additionally, a sensitivity analysis was conducted to determine the stability of the pooled results. The statistical analyses were conducted using Stata 12.0 software (Stata Corp LP, College Station, TX, USA). All P-values were two-sided, and $\mathrm{P}<0.05$ was considered to indicate statistically significant differences.

\section{Results}

Selection and characteristics of studies. A total of 170 studies on the association of OPN with hematological tumors were identified during the initial literature search; 150 studies were excluded after screening the titles or abstracts, as they were either review articles, abstracts, experimental researches, duplicate reports or studies irrelevant to the present analysis. A total of 20 studies were selected for detailed evaluation, 16 of which were excluded following full assessment (1 study was excluded due to a suspected population overlap and 15 studies lacked necessary data). Following careful evaluation based on the inclusion criteria, 4 eligible studies including a total of 492 patients were finally selected for the metaanalysis $(11,12,14)$; the study performed by Liersch et al consisted of three independent researches. Two of the studies were performed in Germany (12), one in Chinese Taiwan (14) and one in Australia (11). The specimen size in each study varied from 52 to 261 patients and the median age of the patients ranged from 45 to 58 years. The percentage of the female population ranged from 37 to $45 \%$. One study used ELISA to measure OPN expression, whereas the others used RT-qPCR, microarray analysis and IHC. OS was reported in each study. The grade of study quality as assessed by NOS ranked from 5 to 7 (Table I). The OPN cut-off value was determined using 
Table III. Characteristics of eligible studies in the metaanalysis on the prognostic value of osteopontin for overall survival in hematological malignancies.

\begin{tabular}{lllr}
\hline Study & $\begin{array}{c}\text { Hazard } \\
\text { ratio }\end{array}$ & $\begin{array}{c}\text { 95\% confidence } \\
\text { interval }\end{array}$ & (Refs.) \\
\hline Liersch et $_{\text {al }}{ }^{\mathrm{a}}$ & 2.35 & $1.09-5.09$ & $(12)$ \\
Liersch et $^{\mathrm{a}}$ & 1.708 & $1.141-2.557$ & $(12)$ \\
Powell et al & 2.09 & $1.16-4.22$ & $(11)$ \\
Lee et al & 2.99 & $1.06-8.44$ & $(14)$ \\
\hline
\end{tabular}

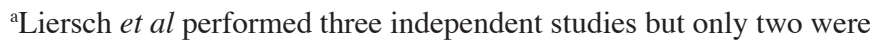
selected for inclusion.

different methods in each study. The basic characteristics of the selected studies are summarized in Table II.

$O P N$ expression and $O S$ in $A M L$. All the studies reported data on OPN expression and OS, as shown Table III. OPN expression was found to be inversely correlated with OS in all studies, and the difference was statistically significant, with a pooled HR estimate of 1.83 (95\% CI: 1.43-2.35, P<0.001, fixed-effects model). There was no significant heterogeneity $\left(\mathrm{I}^{2}=0 \%, \mathrm{P}=0.687\right.$; Fig. 2$)$.

Publication bias. Publication bias assessment was used to evaluate the reliability of the meta-analysis results, particularly those that were found to be statistically significant (15). Publication bias was assessed using the Egger's test (16), with statistical significance set at $\mathrm{P}<0.05$. There was no significant publication bias in the OS studies $(\mathrm{P}=0.687)$.

\section{Discussion}

The clinical outcome in patients with AML depends on various prognostic factors, including patient age and status, as well as the cytogenetic and molecular characteristics of the leukemic clone. Although there have been advances in the diagnosis and treatment, the overall prognosis of AMLs is dismal, due to the poor response to adjunctive treatments and high incidence of tumor recurrence in advanced stages (17). Thus, it is crucial to identify effective biomarkers of survival to help clinical decision-making for AML treatment and improve outcomes. OPN is considered to be one of the candidate markers of stage and prognosis for a number of malignant tumors and has been extensively investigated in that context; however, the available data have not been comprehensively analyzed to date. As meta-analysis is considered to be a valuable method for biomarker validation, the present meta-analysis was performed to investigate the correlation of OPN with AML prognosis.

In the present study, the correlation between OPN overexpression and OS in AML was initially assessed. The pooled results demonstrated that high OPN expression was significantly associated with a poor OS for AML $(\mathrm{P}<0.001)$; Although the analysis outcomes were considered significant, the cause of the differences in OS among AML patients with different OPN expression remain obscure. Several data demonstrated 


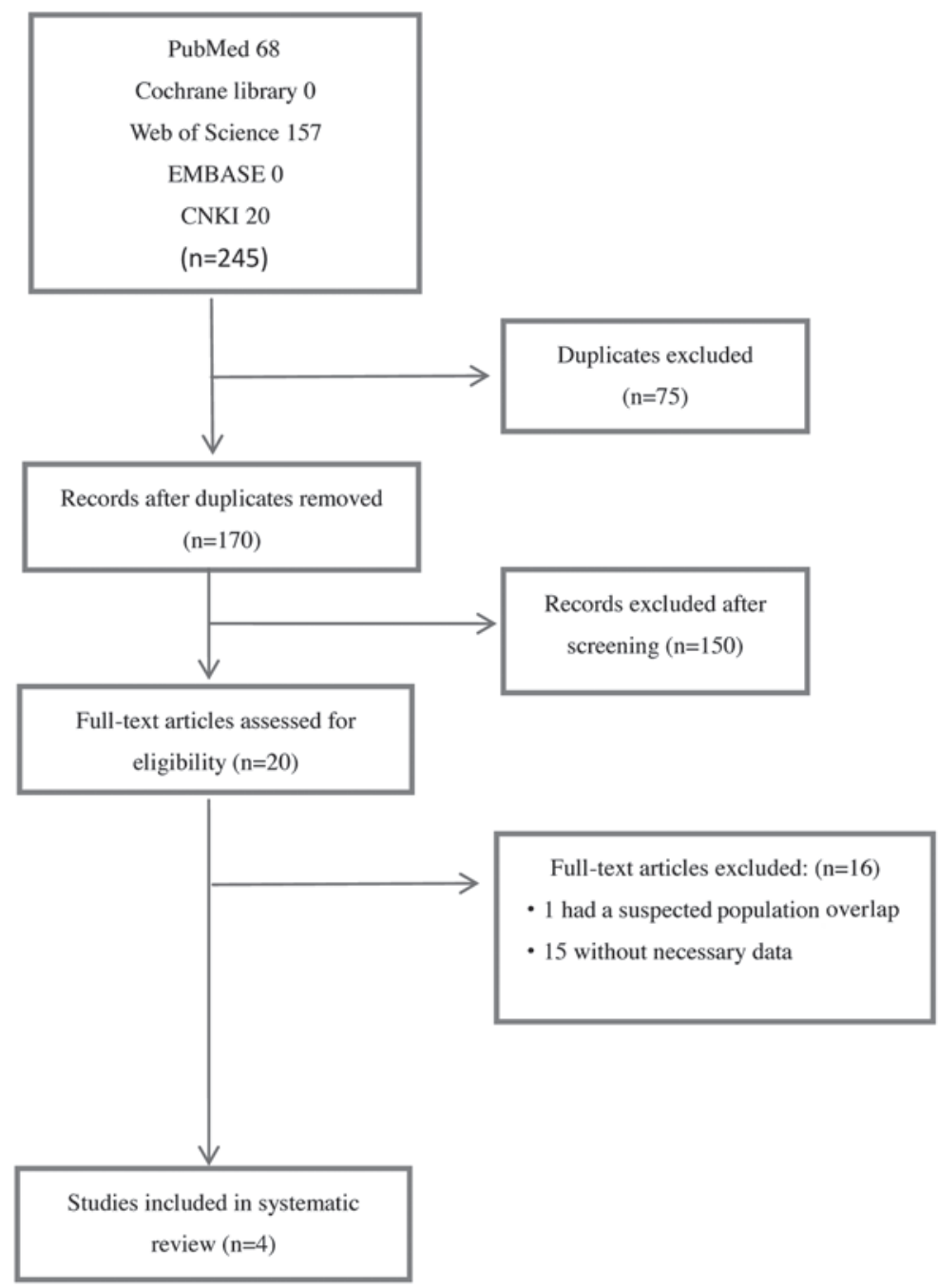

Figure 1. Flow diagram summarizing the identification process of relevant studies. CNKI, China National Knowledge Infrastructure.

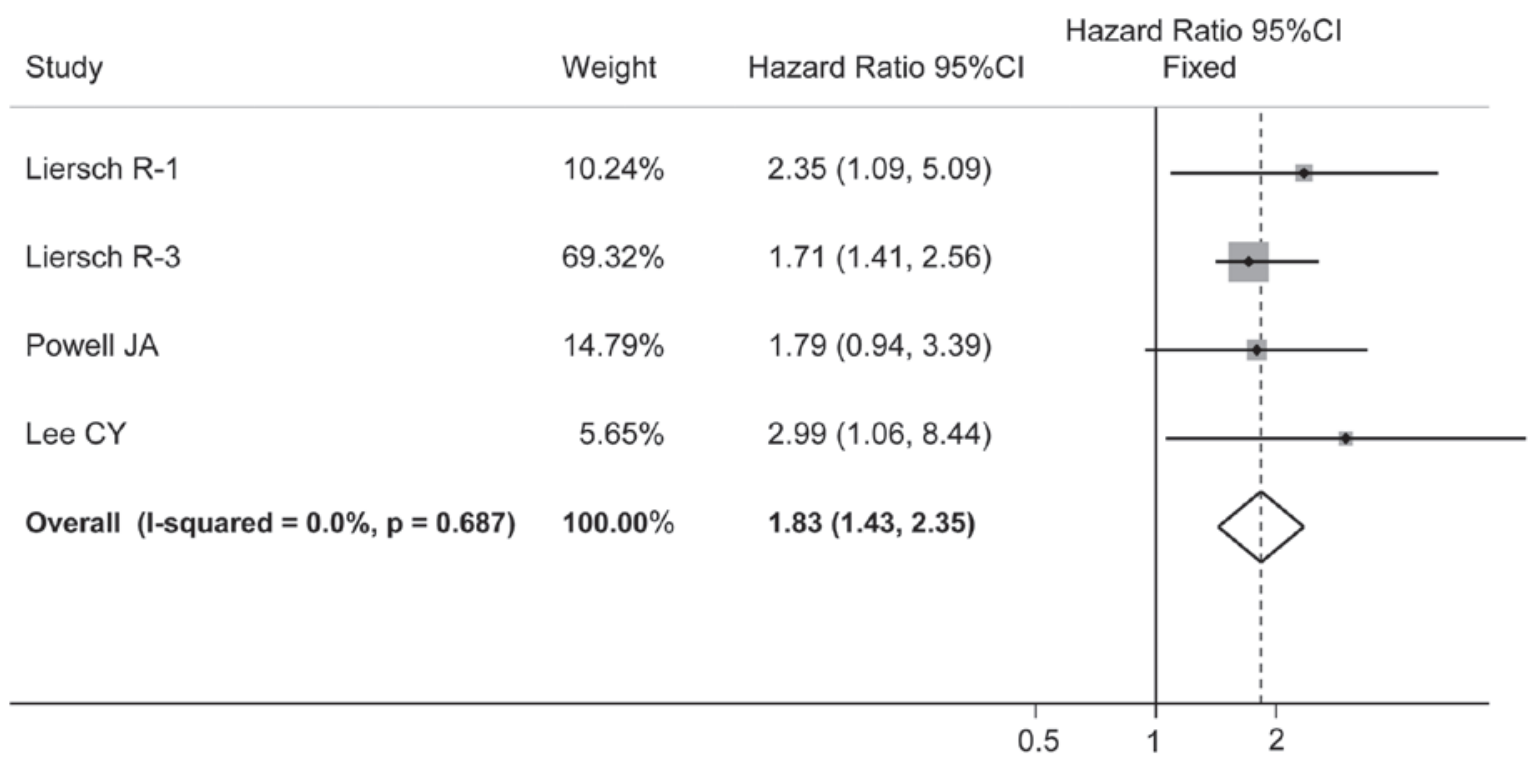

Heterogeneity: $\mathrm{Chi}^{2}=1.48, \mathrm{df}=3(\mathrm{P}=0.687) ; \mathrm{I}^{2}=0 \%$

Test for overall effect: $Z=4.82(P<0.001)$

Figure 2. Forest plot of the association between OPN elevation and OS of patients with hematological malignancies. OPN expression was found to be inversely correlated with OS in all studies, and the difference was statistically significant, with a pooled HR estimate of 1.83 . There was no significant heterogeneity, OPN, osteopontin; OS, overall survival; HR, hazard ratio. 
that OPN expression was inversely correlated with prognosis in AML patients $(11,12,14)$. Whereas the majority of the publications had focused on solid tumors (18-21), there have been recent studies on the role of OPN in hematological malignancies $(10,12)$. Increased serum OPN concentrations have been reported in chronic myeloid leukemia, multiple myeloma and AML (22). Lee et al suggested that higher bone marrow levels of OPN were significantly associated with shorter survival in a 1-year survival analysis of a small cohort of AML patients (14). Powell et al performed expression screening to identify targets in AML (11). These analyses identified OPN as a functionally relevant target. Liersch et al performed three independent cohort studies and identified OPN expression at the mRNA and protein level as a prognostic marker in AML (12). Furthermore, high OPN expression was found to be independently correlated with vascular invasion $(14,23,24)$ and plays an important role in metastasis and stage of hematological malignances $(23,25-27)$, possibly through activation of the mitogen-activated protein kinase and NF- $\kappa \mathrm{B}$ pathways, as well as matrix metalloproteinase-2 (28). In summary, the pooled results of the present meta-analysis supported the hypothesis that OPN overexpression may promote AML progression through direct or indirect mechanisms, leading to a poor outcome of AML.

Although the prognostic role of OPN in AML was comprehensively evaluated, there were certain limitations that should be discussed. First, language and risk bias may be a factor in this meta-analysis, as positive results were more often published compared with negative results; furthermore, only reports written in English or Chinese were selected, which may affect the validity of the results to a certain degree. Second, despite the lack of significant heterogeneity or publication bias, only data from small databases were collected and the number and sample of included studies were relatively insufficient, which may also affect the reliability of the analysis results. Third, geographical bias was a concern. Fourth, as our meta-analysis was performed on the collected data, strong recommendations at an individual patient level could not be obtained. Due to these limitations, the results of the present meta-analysis should be interpreted carefully and conclusions should be drawn with caution.

In summary, OPN overexpression was found to be associated with poor prognosis of AML patients. Therefore, OPN, alone or combined with other markers, may provide important predictive information on AML invasion, metastasis and prognosis and it may also serve as a valuable molecular target for the clinical treatment of AML.

\section{Acknowledgements}

This study was supported by grants from the National Natural Science Foundation of China (no. 81641006) and Beijing City Youth Top-Notch Talents Project (no. 2016000021223ZK16).

\section{References}

1. Yanada M, Narimatsu H, Suzuki T, Matsuo K and Naoe T: Randomized controlled trials of treatments for hematologic malignancies: Study characteristics and outcomes. Cancer 110 334-339, 2007.
2. Burger JA and Peled A: CXCR4 antagonists: Targeting the microenvironment in leukemia and other cancers. Leukemia 23: 43-52, 2009.

3. Dankbar B, Padro T, Leo R, Feldmann B, Kropff M, Mesters RM, Serve H, Berdel WE and Kienast J: Vascular endothelial growth factor and interleukin-6 in paracrine tumor-stromal cell interactions in multiple myeloma. Blood 95: 2630-2636, 2000.

4. Schliemann C, Bieker R, Padro T, Kessler T, Hintelmann H, Buchner T, Berdel WE and Mesters RM: Expression of angiopoietins and their receptor Tie 2 in the bone marrow of patients with acute myeloid leukemia. Haematologica 91: 1203-1211, 2006.

5. Colombo M, Mirandola L, Platonova N, Apicella L, Basile A, Figueroa AJ, Cobos E, Chiriva-Internati M and Chiaramonte R: Notch-directed microenvironment reprogramming in myeloma: A single path to multiple outcomes. Leukemia 27: 1009-1018, 2013.

6. Senger DR, Wirth DF and Hynes RO: Transformed mammalian cells secrete specific proteins and phosphoproteins. Cell 16: 885-893, 1979.

7. Brown LF, Papadopoulos-Sergiou A, Berse B, Manseau EJ, Tognazzi K, Perruzzi CA, Dvorak HF and Senger DR: Osteopontin expression and distribution in human carcinomas. Am J Pathol 145: 610-623, 1994.

8. Agrawal D, Chen T, Irby R, Quackenbush J, Chambers AF, Szabo M, Cantor A, Coppola D and Yeatman TJ: Osteopontin identified as lead marker of colon cancer progression, using pooled sample expression profiling. J Natl Cancer Inst 94: 513-521, 2002.

9. Coppola D, Szabo M, Boulware D, Muraca P, Alsarraj M, Chambers AF and Yeatman TJ: Correlation of osteopontin protein expression and pathological stage across a wide variety of tumor histologies. Clin Cancer Res 10: 184-190, 2004.

10. Chagan-Yasutan H, Tsukasaki K, Takahashi Y, Oguma S, Harigae $\mathrm{H}$, Ishii $\mathrm{N}$, Zhang J, Fukumoto $\mathrm{M}$ and Hattori T: Involvement of osteopontin and its signaling molecule CD44 in clinicopathological features of adult $\mathrm{T}$ cell leukemia. Leuk Res 35: 1484-1490, 2011.

11. Powell JA, Thomas D, Barry EF, Kok CH, McClure BJ, Tsykin A, To LB, Brown A, Lewis ID, Herbert K, et al: Expression profiling of a hemopoietic cell survival transcriptome implicates osteopontin as a functional prognostic factor in AML. Blood 114: 4859-4870, 2009.

12. Liersch R, Gerss J, Schliemann C, Bayer M, Schwöppe C, Biermann C, Appelmann I, Kessler T, Löwenberg B, Büchner T, et al: Osteopontin is a prognostic factor for survival of acute myeloid leukemia patients. Blood 119: 5215-5220, 2012.

13. Stang A: Critical evaluation of the Newcastle-Ottawa scale for the assessment of the quality of nonrandomized studies in meta-analyses. Eur J Epidemiol 25: 603-605, 2010.

14. Lee CY, Tien HF, Hou HA, Chou WC and Lin LI: Marrow osteopontin level as a prognostic factor in acute myeloid leukaemia. $\mathrm{Br}$ J Haematol 141: 736-739, 2008.

15. E Y, He N, Wang Y and Fan H: Percutaneous transluminal angioplasty (PTA) alone versus PTA with balloon-expandable stent placement for short-segment femoropopliteal artery disease: A metaanalysis of randomized trials. J Vasc Interv Radiol 19: 499-503, 2008.

16. Egger M, Davey Smith G, Schneider M and Minder C: Bias in meta-analysis detected by a simple, graphical test. BMJ 315: 629-634, 1997.

17. Kadia TM, Ravandi F, O'Brien S, Cortes J, Kantarjian HM: Progress in acute myeloid leukemia. Clin Lymphoma Myeloma Leuk 15: 139-151, 2015.

18. Zhao M, Liang F, Zhang B, Yan W and Zhang J: The impact of osteopontin on prognosis and clinicopathology of colorectal cancer patients: A systematic meta-analysis. Sci Rep 5: 12713, 2015.

19. Li S, Zou D, Li C, Meng H, Sui W, Feng S, Cheng T, Zhai Q and Qiu L: Targeting stem cell niche can protect hematopoietic stem cells from chemotherapy and G-CSF treatment. Stem Cell Res Ther 6: 175, 2015.

20. Hu ZD, Wei TT, Yang M, Ma N, Tang QQ, Qin BD, Fu HT and Zhong RQ: Diagnostic value of osteopontin in ovarian cancer: A meta-analysis and systematic review. PLoS One 10: e0126444, 2015.

21. Wan HG, Xu H, Gu YM, Wang H, Xu W and Zu MH: Comparison osteopontin vs AFP for the diagnosis of HCC: A meta-analysis. Clin Res Hepatol Gastroenterol 38: 706-714, 2014. 
22. Saeki Y, Mima T, Ishii T, Ogata A, Kobayashi H, Ohshima S, Ishida T, Tabunoki Y, Kitayama H, Mizuki M, et al: Enhanced production of osteopontin in multiple myeloma: Clinical and pathogenic implications. Br J Haematol 123: 263-270, 2003.

23. Wang HL, Ruan LH and Zhao XQ: Expression of osteopontin and VEGF in acute leukemia and their relationship with angiogenesis. Zhongguo Shi Yan Xue Ye Xue Za Zhi 19: 926-929, 2011 (In Chinese)

24. Colla S, Morandi F, Lazzaretti M, Rizzato R, Lunghi P, Bonomini S, Mancini C, Pedrazzoni M, Crugnola M, Rizzoli V and Giuliani N: Human myeloma cells express the bone regulating gene Runx2/Cbfa1 and produce osteopontin that is involved in angiogenesis in multiple myeloma patients. Leukemia 19 2166-2176, 2005

25. Minarik J,Pika T, Bacovsky J,Petrova P, Langova K and Scudla V: Prognostic value of hepatocyte growth factor, syndecan-1, and osteopontin in multiple myeloma and monoclonal gammopathy of undetermined significance. ScientificWorldJournal 2012: 356128, 2012.

26. Sfiridaki A, Miyakis S, Pappa C, Tsirakis G, Alegakis A, Kotsis V, Stathopoulos E and Alexandrakis M: Circulating osteopontin: A dual marker of bone destruction and angiogenesis in patients with multiple myeloma. J Hematol Oncol 4: 22, 2011.
27. Huang Z, Xiao B, Chen S, Li S, Liu Y and Liu J: Studies of WT1 gene expression in leukemia patients. Zhonghua Xue Ye Xue Za Zhi 23: 367-369, 2002

28. Scatena M, Almeida M, Chaisson ML, Fausto N, Nicosia RF and Giachelli CM: NF-kappaB mediates alphavbeta3 integrin-induced endothelial cell survival. J Cell Biol 141: 1083-1093, 1998 